\title{
Nimotuzumab with Induction Chemotherapy and Chemo-Radiation in Patients with Advanced Head and Neck Cancer
}

\author{
Sundaram Subramanium ${ }^{*}$, Venkatanarayan Balasundaram ${ }^{2}$, Sridharan Nithya ${ }^{1}$, \\ Poojar Kiran ${ }^{3}$ \\ ${ }^{1}$ Medical Oncology, V. S. Hospital, Chennai, India \\ ${ }^{2}$ Radiation Oncology, V. S. Hospital, Chennai, India \\ ${ }^{3}$ Clinical Pharmacology, Biocon, Bangalore, India \\ Email: ${ }^{\text {drsundarams7@gmail.com }}$
}

Received 6 January 2015; accepted 29 January 2015; published 30 January 2015

Copyright (C) 2015 by authors and Scientific Research Publishing Inc.

This work is licensed under the Creative Commons Attribution International License (CC BY). http://creativecommons.org/licenses/by/4.0/

(c) (7) Open Access

\section{Abstract}

Background: Head and neck squamous cell carcinoma (HNSCC), is a common malignancy in the Indian Population. In locally advanced disease, chemoradiation is the standard of care. Although induction chemotherapy has been much studied, no clear benefit has been identified apart from laryngeal preservation. A few randomized trials have demonstrated improved response rate, disease free survival, and overall survival, with induction chemotherapy. Nimotuzumab is a humanized monoclonal antibody targeting epidermal growth factor receptors (EGFR). Unlike other AntiEGFR monoclonal antibodies, it is demonstrated to be safer when combined with chemotherapy and/or radiotherapy. Aim: To evaluate the safety and efficacy of concurrently administered nimotuzumab with chemo-radiotherapy in patients with HNSCC in usual health care setting. Methods: This was an open-label, single arm study, with retrospective analysis of results. Patients above 18 years of age, and having histologically confirmed, advanced HNSCC were included in the study. The patients were treated with 3 cycles of induction chemotherapy consisting of modified TPF regimen along with nimotuzumab (200 mg IV) on Day 1, followed by radiotherapy for a dose of 66 Gy along with concurrent weekly cisplatin $\left(30 \mathrm{mg} / \mathrm{m}^{2}\right)$ and nimotuzumab $(200 \mathrm{mg})$ throughout the course of radiation. Patients were evaluated using RECIST criteria, 4 weeks after the last cycle of chemotherapy. Results: Sixteen patients were included in this study, with mean age of $54 \pm 11$ years. Most common sub-site of cancer was oral cavity in $69 \%(n=11)$, followed by pharynx in $19 \%(n=3)$. Four patients had metastasis at the time of presentation. Six patients $(37.5 \%)$ had progressive disease and four patients $(25 \%)$ were lost to follow-up. The combination chemotherapy with nimotuzumab was well tolerated. Addition of nimotuzumab to TPF regimen was not associated with added toxicity. Conclusion: Addition of anti-EGFR monocloncal antibody (nimotuzumab) to induc-

\footnotetext{
${ }^{*}$ Corresponding author.
}

How to cite this paper: Subramanium, S., Balasundaram, V., Nithya, S. and Kiran, P. (2015) Nimotuzumab with Induction Chemotherapy and Chemo-Radiation in Patients with Advanced Head and Neck Cancer. Journal of Cancer Therapy, 6, 146152. http://dx.doi.org/10.4236/jct.2015.62016 
tion chemotherapy and chemoradiation may be a promising alternative to concurrent chemoradiotherapy in HNSCC due to known over expression of EGFR receptors. The results of this study need further evaluation in a larger study setting.

\title{
Keywords
}

\author{
Nimotuzumab, Head and Neck Cancer, Chemoradiotherapy, Cisplatin, Epidermal Growth Factor \\ Receptors, Monoclonal Antibody
}

\section{Introduction}

Head and neck cancers (HNCs) are very common in India, and account for almost 30\% of all cancers in India. Nearly 58\% of the global HNCs occur only in Asia and constitute approximately $5 \%$ of all cancers globally [1] [2]. Squamous cell carcinoma (HNSCC), the most frequent variety of HNC, is a potentially curable malignancy, when diagnosed at an early stage. However, advanced locoregional disease (non-metastatic Stage III or Stage IV) is the most frequent presentation at diagnosis-in almost 60\% of HNSCC patients, and is associated with poor prognosis [3].

The high frequency of HNC in India can be attributed to widespread tobacco exposure, either by smoking or chewing. It accounts for nearly $50 \%$ of oral and oropharyngeal tumours in men and over $90 \%$ cases in women. Other associated risk factors include ionizing radiation, diesel exhausts, sulphuric acid mists, and mustard gas [4].

The standard treatment for patients with operable HNSCC is surgery followed by post-operative radiotherapy (RT), with or without adjuvant chemotherapy, whereas in unresectable locoregionally advanced HNSCC, the approach is concurrent chemoradiotherapy (CTRT) [5]. Induction chemotherapy has a proven role in organ preservation and in some cancers reduced the incidence of distant metastases but its efficacy in prolonging overall survival is yet to be established [5].

Induction chemotherapy with cisplatin and fluorouracil (PF) has shown benefit in locally advanced head and neck cancer by reducing tumour size and micrometastases before definitive radiotherapy. In this long-term analysis of TAX 324, with a median follow-up of 6 years, overall and progression-free survival was significantly better in patients treated with TPF than in those treated with PF (panel). Patients with hypopharyngeal and laryngeal cancer also had significantly longer progression-free survival with TPF than with PF, and those with oropharyngeal primary tumours in the TPF group had an overall survival advantage. Several studies like this observed sustained superiority of docetaxel, cisplatin, and fluorouracil (TPF) over cisplatin and fluorouracil (PF) solidifies the role of TPF as induction chemotherapy for patients with locally advanced squamous-cell carcinoma of the head and neck [6].

Study by Vermorken et al., evaluated docetaxel based regimen (docetaxel, platin, flurouracil) that showed significantly improved progression free and overall survival in patients with unresectable squamous-cell carcinoma of the head and neck were observed with addition of docetaxel in the standard regimen of cisplatin and fluorouracil, induction chemotherapy. Though efficacy was improved, Grade 3/Grade 4 toxicities and increase death rate due to toxicities were the major limitation [7].

Epidermal growth factor receptor (EGFR) is over-expressed in more than $90 \%$ of the HNSCC tumours and is associated with poorer survival [4]. Cetuximab (chimeric MAb) and nimotuzumab (humanized MAb) are the two monoclonal antibodies that are approved in India for the management of advanced head and neck cancer.

Enhancements in survival with the addition of the taxane (docetaxel) to the traditional induction regimen consisting of cisplatin and 5 flurouracil compared with cisplatin and 5 flurouracil alone [8]-[10]. Toxicities associated with this regimen is main limitation, addition of EGFR antibodies not only improve the efficacy but also decreases the toxicities associated [11] [12]. Improved complete response rate was observed when cetuximab added to induction chemotherapy, radiotherapy in patients with locally advanced squamous cell carcinoma of the head and neck [11]. Kies et al., reported enhanced progression-free survival (PFS) and overall survival (OS) rates in patients with advanced nodal disease, common toxicities found with cetuximab were grade 3 or 4 toxicity was skin rash, neutropenia [13]. 
Nimotuzumab (also known as h-R3), is a humanized anti-EGFR monoclonal antibody, what has demonstrated significant clinical and survival benefits with a good safety profile in combination with CTRT/RT [14]-[16]. Babu et al. reported that addition of biologically active targeted therapies to RT/CTRT increase therapeutic outcome with minimal morbidity. Study conducted 76 evaluable patients with (Stage III or IVa), inoperable SCCHN showed that overall survival rate increased when nimotuzumab was added to chemotherapy and radiotherapy and it also significantly reduced risk of death [14].

This study was planned to further evaluate the safety and efficacy of addition of nimotuzumab to induction chemotherapy and concurrent chemoradiotherapy in patients with locally advanced inoperable HNSCC.

\section{Methods}

This was an open label single arm study. Sixteen patients above the age of 18 years, having histologically confirmed HNSCC, in an inoperable stage (III and IV), were enrolled. Other main inclusion criteria were Eastern Cooperative Oncology Group (ECOG) Performance Status score of $\leq 2$ and life expectancy greater than 6 months. The main exclusion criteria were patients who received chemotherapy, radiotherapy and/or immunotherapy, patients with distant metastases and known or suspected hypersensitivity to drugs used in the study.

Informed consent was obtained from all patients. During the screening, 3 cycles of induction chemotherapy consisting of TPF regimen (Paclitaxel $260 \mathrm{mg}$, Cisplatin $30 \mathrm{mg} / \mathrm{m}^{2}$ for 3 days, 5-flurouracil $1500 \mathrm{mg}$ for 3 days and Leucovorin $300 \mathrm{mg}$ IV) along with Nimotuzumab (200 mg IV) on Day 1.

The patients were administered IV cisplatin $30 \mathrm{mg} / \mathrm{m}^{2}$ and IV nimotuzumab $200 \mathrm{mg}$ weekly for 6 weeks, along with radiotherapy of 6600 cGy over 33 fractions. Patients were evaluated over Response Evaluation Criteria in Solid Tumors (RECIST) criteria 24 weeks after the last cycle of chemotherapy.

\section{Results}

\section{Demographics}

Mean age of patients was 54 years, with majority of them (69\%) being males (Table 1). Most common site of cancer was oral cavity (69\%), followed by pharynx (19\%) and larynx (13\%) (Table 2).

All patients completed 3 cycles of induction chemotherapy with TPF regimen and nimotuzumab. Thirteen (81.2\%) patients completed six cycles of concurrent chemoradiotherapy with cisplatin (Figure 1).

Table 1. Distribution of patients with respect to gender and age.

\begin{tabular}{ccc}
\hline Description & Category & Results \\
\hline \multirow{2}{*}{ Gender } & Female & $5(31 \%)$ \\
& Male & $11(69 \%)$ \\
& $\mathrm{N}$ & 16 \\
Age & Mean & 54 \\
(Years) & Standard Deviation & 11 \\
& Median & 57 \\
& (Min., Max.) & $(39,70)$ \\
\hline
\end{tabular}

Table 2. Distribution of patients with respect to tumour location.

\begin{tabular}{ccc}
\hline Tumour Location & No. of Subjects & Frequency \\
\hline Oropharynx & 11 & $69 \%$ \\
Pharynx & 3 & $19 \%$ \\
Larynx & 2 & $13 \%$ \\
Total & 16 & $100 \%$ \\
\hline
\end{tabular}




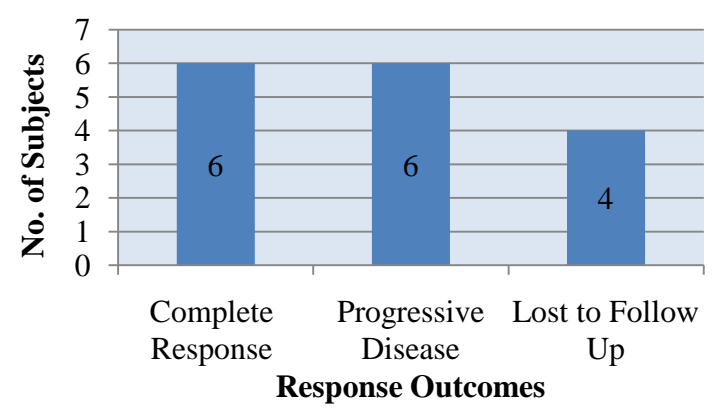

Figure 1. Response rate with nimotuzumab with Induction Chemotherapy and Chemoradiation.

Six patients achieved complete response and are disease free. The median duration of response was 42 months. (21 - 73 months), the patient and the tumor characteristics of these patients are detailed in Table 3.

All the patients tolerated therapy well; no adverse events or toxicities were observed in any of the treated patients. Addition of nimotuzumab to the TPF regimen was not associated with added toxicity.

\section{Discussion}

Induction chemotherapy is planned based on the hypothesis that it would involve a better drug delivery in well vascularised tumours and could eliminate the micrometastatic disease with active doses of chemotherapy given systemically [12].

Induction regimens including fluorouracil and platins (PF) demonstrated organ preservation, but long term survival benefits were seen only in few trials. Incorporation of taxanes (TPF) to the two drug regimens has shown an increase in the response rates (higher than $90 \%$ and complete responses in more than $50 \%$ of patients) and survival benefit. Study by Vermorken et al., evaluated docetaxel based regimen (docetaxel, platin, flurtouracil) that showed a significant higher progression free survival (PFS) and overall survival (OS) compared to PF regimen (PFS: 11.0 months vs. 8.2 months; OS was 18.8 months vs. 14.5 months) [12].

Induction therapy showed improved overall survival. Two important studies TAX 324, TAX 323 confirm it but this approach had disadvantage of toxicity. Main findings of the TAX 324 study conducted with two group PF $(\mathrm{N}=246)$, TPF $(\mathrm{N}=255)$ was overall response after induction chemotherapy was $64 \%$ in PF group versus $72 \%$ in TPF group, complete responses in PF group was 15\% and 17\% in TPF group. Enhanced median overall survival was 71 months in TPF group than 30 months in PF group, significant improvement in PFS at 3 years (TPF 53 vs. PF 42). Enhanced overall survival at 3 years was (42 PF group versus 53 in TPF group). Total deaths was 54 in PF group and in TPF group 41. Toxicities like neutropenia was observed in more than 50\% patients (in TPF group 83\% patients observed neutropenia) other toxicities associated was febrile neutropenia/neutropenic infection, anemia, thrombocytopenia, stomatitis [6]. Similar results observed in TAX 323 like significant higher progression free survival (PFS) and overall survival (OS) compared to PF regimen (PFS: 11.0 months vs. 8.2 months; OS was 18.8 months vs. 14.5 months) [7].

Elevated levels of EGFR are associated with malignant transformation of neoplastic cells and its role in causing radioresistance by mediating cell survival following radiotherapy in cells has been studied [9] [17]-[19]. The anti-EGFR agents have shown to inhibit the EGFR-signaling pathway and reduce the rate of cellular proliferation, and also negate the EGFR-mediated radioresistance and sensitize malignant cells to concomitant RT [18] [19]. Similarly, EGFR antagonists may augment the tumour response to chemotherapy since EGFR overexpression is also known to cause resistance to chemotherapeutic agents [19]. Thus, the use of EGFR inhibitors may enhance tumour cell killing by limiting the free radical-induced AKT-mediated cell survival [10]. Cetuximab (chimeric $\mathrm{MAb}$ ) and nimotuzumab (humanized $\mathrm{MAb}$ ) are the two monoclonal antibodies that are approved in India for the management of advanced head and neck cancer.

Tremplin study conducted in 98 intent to treat population of which 52 patients (86\%) were included in Arm A RT (70 Gy) with cisplatin (100 mg/m² on Days 1, 22 and 43 of RT) and 46 pts (82\%) in Arm B RT (70 Gy) with cetuximab (400 mg/m² loading dose before RT and $250 \mathrm{mg} / \mathrm{m}^{2}$ on the first day of the 7 weeks of RT). Overall survival was respectively $85 \%$ and $86 \%$ in Arm A vs. Arm B. Local failures occurred more often in Arm B, better patient compliance with Arm B. Acute and late toxicities was higher in Arm A [20]. In another study Mesia et al., 
Table 3. Patient characteristics and survival of patients with complete response to therapy.

\begin{tabular}{ccccc}
\hline Age/Sex & Location of Cancer & Tnm Staging & Date of Diagnosis & Last Day of Follow up \\
\hline 47/F & Hypopharynx & T3N2M0 & $08 / 11 / 2010$ & $18 / 10 / 2013$ \\
42/M & Tongue & T3N2M0 & $09 / 05 / 2011$ & $06 / 03 / 2013$ \\
56/F & Larynx & T3N2M0 & $22 / 11 / 2006$ & $20 / 09 / 2013$ \\
40/M & Tongue & T4aN1M0 & $21 / 02 / 2008$ & $04 / 04 / 2013$ \\
70/M & Left Tonsil & T3N2bM0 & $18 / 04 / 2010$ & $05 / 05 / 2011$ \\
37/M & Tongue & T2N2M0 & $14 / 08 / 2009$ & $24 / 11 / 2012$ \\
\hline
\end{tabular}

showed that adding cetuximab to PF chemotherapy was superior to PF alone in unresectable SCCHN. Phase II trial conducted in 7 Spanish hospitals. Induction comprised TPF, cetuximab and accelerated RT with a concomitant boost (69.9 Gy). Complete response was observed in 6 patients (12\%) after two cycles and in 10 patients (20\%) after four cycles. Thirty-one patients (62\%) showed partial response after two cycles while after for cycles in 25 patients (50\%) showed partial response. After two cycles 5 patients (10\%) showed stable disease and 4 patients (8\%) was in stable disease stage after four cycles. Progressive disease was in 1 patient (2\%) and 2 patients (4\%) after two cycles and four cycles respectively. However, in this study, 2 adverse event related deaths (febrile neutropenia and hepatic insufficiency) were observed. Other serious Grade 3/4 adverse events (AEs) like neutropenia; neutropenic fever, infection thrombocytopenia, diarrhea, rash, hypotension were also there [21]-[24].

Zhao et al., reported in forty cases of resectable HNSCC were treated with nimotuzumab (400 mg on Day 1) combined with PF regimens (cisplatin $75 \mathrm{mg} / \mathrm{m}^{2}$ on Days 1 and 5-Fu $750 \mathrm{mg} / \mathrm{m}^{2}$ administered on Days 1-5 in a 3 weekly regimen) and after 2 cycles, an organ-preservation local therapy (surgery or radiotherapy) 85\% patients showed complete or partial response. Many of the patient showed downstaging, nimotuzumab found to be highly effective for preserving the organ function and the toxicities are well tolerable [13]. Rodriguez et al., observed complete response after treatment with paclitaxel, nimotuzumab and low-dose methotrexate in a 61-year-old male patient with metastatic poorly differentiated squamous cell carcinoma of larynx to lymph nodes and lung. The toxicity was very mild, only neutropenia Grade I and peripheral neuropathy Grades I-II. There was no evidence of skin rash. The patient was asymptomatic with excellent general condition and without any tumor activity on physical examination [17]. The current approach of use of induction chemotherapy followed by definitive local therapy has the advantage of the potential to reduce the risk of distant failure and a rapid reduction of tumours in the responders. In addition, induction also appears to predict the responsiveness to chemoradiotherapy [12].

Our study showed that addition of nimotuzumab to induction chemotherapy with taxanes, platins and flurouracil (TPF) regimen followed by concurrent CTRT has resulted in complete response CR in 38\% patients, without any significant toxicity. These study results are concurrent with the studies discussed earlier. The safety of nimotuzumab has been repeatedly demonstrated in previous studies [8] [25]-[27]. Unlike other anti-EGFR antibodies, nimotuzumab requires bivalent binding for stable attachment. This leads to selective binding to cells expressing moderate to high EGFR levels, and thereby a better safety profile. In normal tissues, where the EGFR concentration is low, nimotuzumab monovalent interaction is transient, thus avoiding severe toxicities [27]. The limitations of this study include small sample size and incomplete capturing of the survival outcomes. However, we strongly believe that targeted therapies along with induction chemoradiotherapy and concomitant chemoradiation stand to add benefit in terms of tumor response, PFS and overall survival. A larger study would be the way ahead for prove these claims.

\section{Conclusion}

These findings suggest that addition of nimotuzumab to induction chemotherapy may be therapeutic option in patients with inoperable, locally advanced HNSCC. Early diagnosis, along with new promising therapies, will help improve outcomes in these cases. However, long term survival benefits of these therapies need to be explored through large scale well designed clinical studies. 


\section{Competing Interests}

The authors declare that they have no competing interests.

\section{References}

[1] Reuter, C.W., Morgan, M.A. and Eckardt, A. (2007) Targeting EGF-Receptor-Signalling in Squamous Cell Carcinomas of the Head and Neck. British Journal of Cancer, 96, 408-416.

[2] Trivedi, N.P., Kekatpure, V.D., Trivedi, N.N. and Kuriakose, M.A. (2012) Head and Neck Cancer in India: Need to Formulate Uniform National Treatment Guideline? Indian Journal of Cancer, 49, 6-10. http://dx.doi.org/10.4103/0019-509X.98907

[3] Million, R.R. (1992) Cancer of the Head and Neck. In: De Vita Jr., V.T., Hellman, S. and Rosenberg, S.A., Eds., Cancer: Principles and Practice of Oncology, 4th Edition, JB Lippincott, Philadelphia, 96-420.

[4] Barnes, et al. Head and Neck: Squamous Cell Carcinoma: An Overview. Atlas of Genetics and Cytogenetics in Oncology and Haematology. http://atlasgeneticsoncology.org/Tumors/HeadNeckSCCID5090.html

[5] Zorat, P.L. and Paccagnella, A. (2004) Randomized Phase III Trial of Neoadjuvant Chemotherapy in Head and Neck Cancer: 10-Year Follow-Up. Journal of the National Cancer Institute, 96, 1714-1717.

[6] Lorch, J.H., Goloubeva, O., Haddad, R.I., et al. (2011) Induction Chemotherapy with Cisplatin and Fluorouracil Alone or in Combination with Docetaxel in Locally Advanced Squamous-Cell Cancer of the Head and Neck: Long-Term Results of the TAX 324 Randomised Phase 3 Trial. The Lancet Oncology, 12, 153-159. http://dx.doi.org/10.1016/S1470-2045(10)70279-5

[7] Vermorken, J.B., Remenar, E., Herpen, C.V., et al. (2007) Cisplatin, Fluorouracil, and Docetaxel in Unresectable Head and Neck Cancer. The New England Journal of Medicine, 357, 1695-1704. http://dx.doi.org/10.1056/NEJMoa071028

[8] Lorch, J.H., Posner, M.R., Wirth, L.J. and Haddad, R.I. (2008) Induction Chemotherapy in Locally Advanced Head and Neck Cancer: A New Standard of Care? Hematology/Oncology Clinics of North America, 22, 1155-1163. http://dx.doi.org/10.1016/j.hoc.2008.08.004

[9] Posner, M.R., Hershock, D.M., Blajman, C.R., et al. (2007) Cisplatin and Fluorouracil Alone or with Docetaxel in Head and Neck Cancer. The New England Journal of Medicine, 357, 1705-1715. http://dx.doi.org/10.1056/NEJMoa070956

[10] Pointreau, Y., Garaud, P., Chapet, S., et al. (2009) Randomized Trial of Induction Chemotherapy with Cisplatin and 5Fluorouracil with or without Docetaxel for Larynx Preservation. Journal of the National Cancer Institute, 101, 498-506. http://dx.doi.org/10.1093/jnci/djp007

[11] Jessica, B., Corey, L., Harry, Q., et al. (2013) Induction Chemotherapy with Cetuximab, Carboplatin and Paclitaxel for the Treatment of Locally Advanced Squamous Cell Carcinoma of the Head and Neck. Experimental and Therapeutic Medicine, 5, 1247-1253.

[12] Ling, Y., Chen, J., Tao, M., et al. (2012) A Pilot Study of Nimotuzumab Combined with Cisplatin and 5-FU in Patients with Advanced Esophageal Squamous Cell Carcinoma. Journal of Thoracic Disease, 4, 58-62.

[13] Kies, M.S., Holsinger, F.C., Lee, J.J., et al. (2010) Induction Chemotherapy and Cetuximab for Locally Advanced Squamous Cell Carcinoma of the Head and Neck: Results from a Phase II Prospective Trial. Journal of Clinical Oncology, 28, 8-14. http://dx.doi.org/10.1200/JCO.2009.23.0425

[14] Babu, K.G., Viswanath, L., et al. (2010) An Open-Label, Randomized, Study of h-R3mAb (Nimotuzumab) in Patients with Advanced (Stage III or IVa) Squamous Cell Carcinoma of Head and Neck (SCCHN): Four-Year Survival Results from a Phase IIb Study. Journal of Clinical Oncology, 28, 15s, Abstr 5530.

[15] Bianco, C., Tortora, G., et al. (2002) Enhancement of Antitumor Activity of Ionizing Radiation by Combined Treatment with the Selective Epidermal Growth Factor Receptor-Tyrosine Kinase Inhibitor ZD1839 (Iressa). Clinical Cancer Research, 8, 3250-3258.

[16] Rodríguez, M.O., Rivero, T.C., et al. (2010) Nimotuzumab Plus Radiotherapy for Unresectable Squamous-Cell Carcinoma of the Head and Neck. Cancer Biology \& Therapy, 9, 343-349.

[17] Wang, H.Q., Quan, T., et al. (2003) Epidermal Growth Factor Receptor-Dependent, NF-kappa B-Independent Activation of the Phosphatidylinositol 3-Kinase/Akt Pathway Inhibits Ultraviolet irradiation-Induced Caspases-3, -8, and -9 in Human Keratinocytes. The Journal of Biological Chemistry, 278, 45737-45745.

[18] Li, H.F., Kim, J.S. and Waldman, T. (2009) Radiation-Induced Akt Activation Modulates Radioresistance in Human Glioblastoma Cells. Radiation Oncology, 4, 43.

[19] Nagane, M., Levitzki, A., Gazit, A., Cavenee, W.K. and Huang, H.J. (1998) Drug Resistance of Human Glioblastoma Cells Conferred by a Tumor-Specific Mutant Epidermal Growth Factor Receptor through Modulation of Bcl-XL and Caspase-3-Like Proteases. Proceedings of the National Academy of Sciences of the United States of America, 95, 
5724-5729.

[20] Lefebvre, J., Pointreau, Y., Rolland, F., et al. (2011) Sequential Chemoradiotherapy (SCRT) for Larynx Preservation (LP): Results of the Randomized Phase II TREMPLIN Study. Journal of Clinical Oncology, 29, Abstr 5501.

[21] Mesia, R.S., Vazquez, J.J., Grau, J.A., et al. (2009) A Single-Arm Phase II Trial to Evaluate the Combination of Cetuximab Plus Docetaxel, Cisplatin, and 5-Fluorouracil (TPF) as Induction Chemotherapy (IC) in Patients (pts) with Unresectable SCCHN. Journal of Clinical Oncology, 27, 15s, Abstr 6015.

[22] Jain, P., Kumar, P., Pai, V.R. and Parikh, P.M. (2008) Neoadjuvant Chemotherapy or Chemoradiotherapy in Head and Neck Cancer. Indian Journal of Cancer, 45, 83-89. http://dx.doi.org/10.4103/0019-509X.44061

[23] Zhao, X.Y., Guo, Y., Zhu, Y.X., et al. (2012) Clinical Analysis of Nimotuzumab Plus Cisplatin and Fluorouracil Regimen as Induction Treatment in Resectable Head and Neck Squamous Cell Carcinoma. Chinese Journal of Otorhinolaryngology Head and Neck Surgery, 47, 536-539.

[24] Verduzco-Rodríguez, L., Aguirre-González, E.H. and Verduzco-Aguirre, C. (2011) Durable Complete Response Induced by Paclitaxel-Nimotuzumab-Methotrexate Chemotherapy in a Patient with Metastatic Head and Neck Squamous Cell Carcinoma. Hematology/Oncology and Stem Cell Therapy, 4, 182-184.

[25] Wang. X.T., McCullough, K.D., Franke, T.F. and Holbrook, N.J. (2000) Epidermal Growth Factor Receptor-Dependent Akt Activation by Oxidative Stress Enhances Cell Survival. The Journal of Biological Chemistry, 275, 1462414631.

[26] Rojo, F., Gracias, E., et al. (2010) Pharmacodynamic Trial of Nimotuzumab in Unresectable Squamous Cell Carcinoma of the Head and Neck: A SENDO Foundation Study. Clinical Cancer Research, 16, 2474-2482.

[27] Garrido, G., Tikhomirov, I.A., et al. (2011) Bivalent Binding by Intermediate Affinity of Nimotuzumab: A Contribution to Explain Antibody Clinical Profile. Cancer Biology \& Therapy, 11, 373-382. 
Scientific Research Publishing (SCIRP) is one of the largest Open Access journal publishers. It is currently publishing more than 200 open access, online, peer-reviewed journals covering a wide range of academic disciplines. SCIRP serves the worldwide academic communities and contributes to the progress and application of science with its publication.

Other selected journals from SCIRP are listed as below. Submit your manuscript to us via either submit@scirp.org or Online Submission Portal.
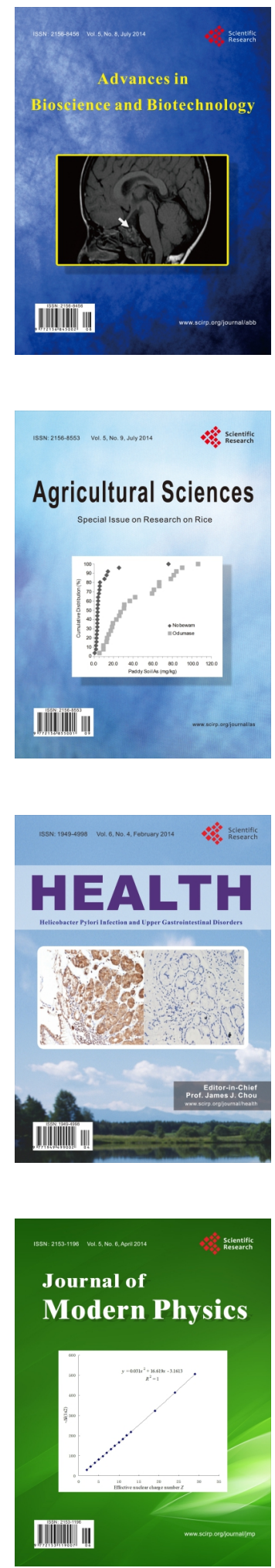
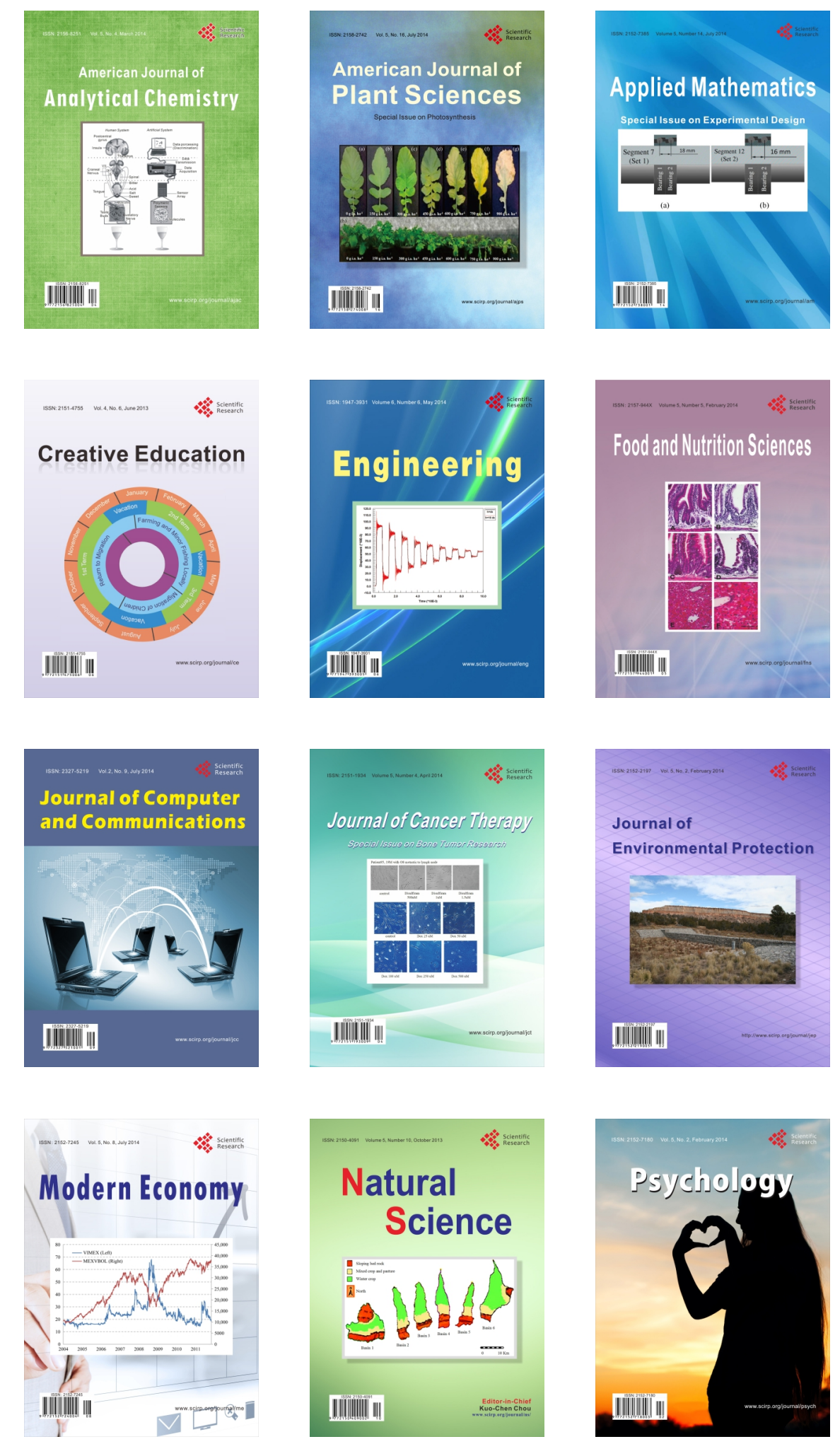